\title{
Die Ärzteeinkommen
}

Während vieler Jahre waren die Ärzteeinkommen ein beliebtes Thema der Massenmedien. Die Bearbeitung dieses Themas erfolgte meistens sehr viel mehr über emotionelle Kanäle als über sachliche Überlegungen.

Noch vor zwanzig Jahren wurden die Ärzteeinkommen in ähnlicher Weise skandalisiert wie heute die Manager-Abgangsentschädigungen und -löhne.

Es wäre ein Einfaches gewesen, die ganze Frage der Ärzteeinkommen auf die Basis von objektiven Erhebungen zu stellen. Ich erinnere daran, dass die Verbindung der Schweizer Ärztinnen und Ärzte seit bald dreissig Jahren die Ärzteeinkommen in einem Rhythmus von zwei Jahren (der Länge einer Wehrsteuerperiode) auf der Basis der AHV-Unterlagen erheben lässt. Die Ärzte als Selbständigerwerbende bezahlen ihre persönlichen AHV-Beiträge auf der Basis ihres Berufs-Netto-Einkommens. Kapitalerträge, Hypothekarzinsen, Erbschaften usw. spielen für die Berechnung des AHV-pflichtigen Einkommens keine Rolle.

Das AHV-pflichtige Einkommen, das den Erhebungen der FMH zugrunde liegt, stellt keine Selbstdeklaration des Arztes dar. Zuerst stellt der Arzt, meistens mit Hilfe eines Treuhänders, seine Steuererklärung auf, wobei hie und da Abgrenzungen mit einem gewissen Spielraum erfolgen. Als zweite Stufe wird die Steuererklärung des Arztes von speziell dafür geschulten Steuerkommissären der kantonalen Wehrsteuerverwaltung überprüft. Diese haben keine Hemmungen, fragliche Positionen der Steuererklärung aufgrund von Erfahrungswerten zu hinterfragen und ohne Gegenbeweis ganz sec mit einer Verfügung abzuändern. Nach solchen allfälligen Korrekturen wird die Steuererklärung von der kantonalen Wehrsteuerverwaltung genehmigt und als richtig erachtet. Die Eckwerte dieser genehmigten Steuererklärung werden der AHV-Ausgleichskasse des Arztes gemeldet, welche nachher aufgrund einer weiteren Prüfung auf Richtigkeit und Plausibilität die vom Arzt geschuldeten persönlichen AHV-Beitrage für die Dauer von zwei aufeinanderfolgenden Jahren festlegt.

$\mathrm{Zu}$ Beginn der FMH-Erhebungen brauchte es jedesmal eine Ausnahmebewilligung des Eidgenössischen Departements des Innern, dass die AHV-Ausgleichskasse von der AHV-Schweigepflicht entbunden wurde und die entpersonalisierten Daten herausgeben durfte.

Inzwischen wurde dieses Procedere um einiges vereinfacht.

Ich stelle fest, dass die Ärzteeinkommen in den letzten drei Jahren wieder an allgemeinem Interesse verloren haben. Ich bin überzeugt, dass dieser Trend $\mathrm{zu}$ einem grossen Teil der offener gewordenen Informationspolitik der FMH zu verdanken ist.

Die Publikation des entsprechenden Artikels in dieser Nummer der Schweizerischen Ärztezeitung und die Tatsache, dass das neue Einkommensgutachten vollständig auf das Internet in der FMH-eigenen homepage (www.fmh.ch über das HIN Health Info Net) aufgeschaltet wird, unterstreicht diese Änderung der FMHInformationspolitik augenfällig.

Möge dies alles dazu dienen, dass die Ärzteeinkommen zwar weiter diskutiert werden können, aber mit der notwendigen Objektivität und emotionsloser Sachlichkeit.

Dr. med. Niklaus Hasler 